\title{
COVID-19 and its Impact for Pregnant Women: A Review
}

\author{
Maria A Grácio* \\ Institute of Hygiene and Tropical Medicine, New University of Lisbon, Lisbon, Portugal \\ ${ }^{\star}$ Corresponding author: Maria A Grácio, Institute of Hygiene and Tropical Medicine, New University of Lisbon, Rua da Junqueira 100, 1348-008 Lisbon, Portugal
}

Received: June 09, 2021; Accepted: June 11, 2021; Published: June 172021

\begin{abstract}
Considering the medical, economic and social importance of the COVID-19 disease in the world, where it is present as indigenous or imported, we have as objectives in this manuscript to contribute to the knowledge of the impact on this viral disease on pregnant women.
\end{abstract}

Keywords: Coronavirus, COVID-19, SARS Coronavirus 2, SARS-Co2, Pregnancy, Obstetrics, Gynecology

\section{Introduction}

COVID-19 is a viral disease whose causative agent was identified in Wuhan-China, as a novel coronavirus, Severe Acute Respiratory Syndrome Coronavirus2 (SARS-CoV-2) [1]. After, 15 April 2020, COVID-19 has caused more than two million confirmed cases and more than 128,000 deaths globally, including 82,295 confirmed cases and 3,342 deaths in China [2]. The Chinese government has locked Wuhan city, since 23 January 2020, and implemented a series of social distancing measures such as: strict traffic restrictions, prohibition of social gatherings; and closure of residential communities [3]. In [4] the authors have referred to "the epidemiological data in China that have shown that most cases had mild symptoms, with an overall case fatality rate of 2,3\%. Although, SARS-CoV-2 appears to be less virulent than 2 previous zoonotic coronavirus, SARS-CoV and Middle East Respiratory Syndrome Coronavirus (MERS-CoV), it is far more efficient in transmitting between people in close contact".

In pregnant women, this novel coronavirus has caused severe complications and both SARS-CoV and MERS-CoV have been found in pregnant women $[5,6]$.

Vertical transmission of this virus has been suspected by several scientists [7], referred to three documented cases of vertical SARSCo-V2 infection, that were accompanied by a strong inflammatory response. Together, this data supports the hypothesis that in utero SARS-Co-V2 vertical transmission while low is possible.

In [8], we have, in our opinion, a good description of the principal symptoms of Coronavirus-19 in humans, and very accessible for scientist and public in general. In that publication, we found the following information: People with COVID-19 have had a wide range of symptoms reported, ranging from mild symptoms to severe illness. Symptoms may appear 2-14 days after exposure to the virus. People with the following symptoms may have COVID-19:

Fever or chills; Cough; Shortness of breath or difficult breathing; Fatigue; Muscle or body aches; Headache; New loss of taste or small; Sore throat; Congestion or runny nose; Nausea or vomiting and Diarrhoea.
In this publication is observed that "the list does not include all possible symptoms. CDC will continue to update this list as we learn more about COVID-19."

Concerning risk for COVID-19 disease, are mentioned: old persons; with chronic diseases: heart diseases, pulmonary, neoplasia or arterial hypertension, Other people cited are with the immunological system compromised undergoing chemotherapy, for auto-immunes diseases (rheumatoid arthritis, lupus, multiple sclerosis, or some inflammatory intestinal diseases), human immunodeficiency virus syndrome, or patients with transplants.

Concerning transmission, the authors in [8], have informed that: the data sources were "eligible studies published until May 28, 2020, were retrieved from PubMed, EMBASE, medRxiv, and bioRxiv". These authors have concluded: "vertical transmission of severe acute respiratory syndrome corona virus 2 is possible and seems to occur in a minority of cases of maternal corona virus disease 2019 infection in the third trimester. The rates of infection are similar to those of other pathogens that cause congenital infections. However, given the paucity of early trimester data, the assessment has yet been made regarding the rates of vertical transmission in early pregnancy and potential risk for consequent fetal morbidity and mortality".

In [9] the authors have presented "An Analysis of 38 Pregnant Women with COVID-19. Their Newborn Infants, and Maternal- Fetal Transmission of SARS-CoV-2: Maternal Coronavirus Infections and Pregnancy Outcomes 4". In their manuscript, they have reviewed the effects of two previous coronavirus infections - Severe Acute Respiratory Syndrome (SARS) caused by SARS-CoV and Middle East Respiratory Syndrome (MERS) caused by MERS-CoV - on pregnancy outcomes. On the other hand "analyses were made of the literature describing 38 pregnant women with COVID-19 and their newborns in China to assess the effects of SARS-CoV-2 on the mothers and infants including clinical, laboratory and virological data, and the transmissibility of the virus from mother to fetus. This analysis reveals that unlike coronavirus infections of pregnant women caused by SARS and MERS, in these 38 pregnant women COVID-19 did not lead to 
maternal deaths. Importantly, and similar to pregnancies with SARS and MERS, there were no confirmed cases of intrauterine transmission of SARS-CoV-2 from mothers with COVID-19 to their fetuses. All neonatal specimens tested, including in some cases placentas, were negative by rt-PCR for SARS-CoV-2. At this point in the global pandemic of COVID-19 infection there is no evidence that SARS [1] CoV-2 undergoes intrauterine or transplacental transmission from infected pregnant women to their fetuses. Analysis of additional cases is necessary to determine if this remains true."

In [10] The authors have referred: (i) that the disease caused by SARS-CoV-2 was named COVID-19 by WHO and has so far killed more people than SARS and MERS; (ii) in January 2020, the World Health Organization declared COVID-19 a pandemic disease, considering, the widespread global outbreak of COVID-19, with more than 132,758 confirmed cases and 4,955 deaths worldwide; (iii) "Research on both SARS-CoV and MERS-CoV, which are pathologically similar to SARS-CoV-2, has shown that being infected with these viruses during pregnancy increases the risk of maternal death, stillbirth, intrauterine growth retardation and, preterm delivery"; (iv) "With the exponential increase in cases of COVID-19 throughout the world, there is a need to understand the effects of SARS-CoV-2 on the health of pregnant women, through extrapolation of earlier studies that have been conducted on pregnant women infected with SARS-CoV, and MERS-CoV. There is an urgent need to understand the chance of vertical transmission of SARSCoV-2 from mother to fetus and the possibility of the virus crossing the placental barrier."

In [11] the authors have referred to the fact that it was their intention "to review published studies related to the association of severe acute respiratory syndrome coronavirus 2 (SARS-Co.2) infections with pregnancy, fetal, and neonatal outcomes during coronavirus disease 2019 (COVID-19) pandemic in a systematic manner." In the methods the authors have indicated that "A comprehensive electronic search was done through PubMed, Scopus, Medline, Cochrane database, and Google Scholar from December 01, 2019, to May 22, 2020, along with the reference list of all included studies. All cohort studies that reported on outcomes of COVID-19 during pregnancy were included. Qualitative assessment of included studies was performed using the Newcastle-Ottawa scale" [12].

The authors have screened 513 titles, and included 22 studies, which identified 156 pregnant women with COVID-19 and 108 neonatal outcomes, and they have concluded that "COVID-19 infection in pregnancy leads to increased risk in pregnancy complications such as preterm birth, PPROM, and may possibly lead to maternal death in rare cases."

\section{Conclusion}

1. We think that it was here demonstrated that COVID-19, has an impact on the health of pregnant women.

2. We hope that with the attention that is being given to this viral disease is possible, in a short/medium time, to obtain more knowledge, concerning the virus, the treatment and the vaccines, so that with this knowledge is possible a control of all viral variants circulating in the world.

3. To combat COVID-19, it is necessary:

- to have persons specialized for the different types of combat;

- the collaboration between countries at world level;

- the collaboration of the person, in general, for the execution of the rules stablished by health services of their countries;

- the collaboration between different governmental services;

- the collaboration between different community services such as town halls and, hospitals.

\section{References}

1. Archived: WHO Timeline - COVID-19, 27 April 2020 - www.who.int

2. World Health Organization. Novel coronavirus - China. Geneva, Switzerland: Word Health Organization httpps.//www.who.int/csr/don/12-january-2020-novelcoronavirus-china/en/. [2020-01-12]

3. COVID-19 - Global Health, COVID-19: What you need to know about the coronavirus pandemic in 15 April. www.weform.org

4. Wuham lockdown: a year of Chinas fight against the COVID pandemic - 22 January, Corona virus pandemic www.bbc.com

5. World Health Organization. The epidemiological characteristics of an outbreak of 2019 novel coronavirus disease (COVID-19)- China 2020.

6. Y Liu, H Chen, K Tang, Y Guo (2020) Clinical manifestations and outcome of SARS$\mathrm{CoV}-2$ infection during pregnancy. The journal of Infection. [crossref]

7. Tseng J-Y (2020) Potential implications of SARS-CoV-2 on pregnancy. Taiwan J Obstet Gynecol 59: 464-465. [crossref]

8. Feuzina C, Biasin M, Catin I, Vergani P, Milet D, Spinilio A, Gismondo M, et al. Analysis of SARS-CoV-2 vertical transmission during pregnancy. Nature Communications 11: 5128. [crossref]

9. Kotlyar A, Grechukona O, Chen A, Shota P, Grimshaw A, et al. (2021) Vertical transmission of corona virus disease 2019: a systematic review and meta-analysis. Am J Obstet Gynecol 224: 35-53.e3. [crossref]

10. Schwartz DA (2020) An Analysis of 38 Pregnant Women with COVID-19, Their Newborn Infants, and Maternal-Fetal Transmission of SARS-CoV-2: Maternal Coronavirus Infections and Pregnancy Outcomes. Arch Pathol Lab Med 144: 799-805. [crossref]

11. Fathi M, Vakili K, Deravi N, Yaghoobpoor S, Ahsan E, et al. (2020) Coronavirus diseases and pregnancy: COVID-19, SARS, and MERS. Przegl Epidemiol 74: 276289. [crossref]

12. Hubba Akhtar, Chandni Patel, Eyad Abuelgasim, Amer Harky (2020) COVID-19 (SARS-CoV-2) Infection in Pregnancy: A Systematic Review. Gynecol Obstet Invest 85: 295-306. [crossref]

\section{Citation:}

